\section{Leading with linen}

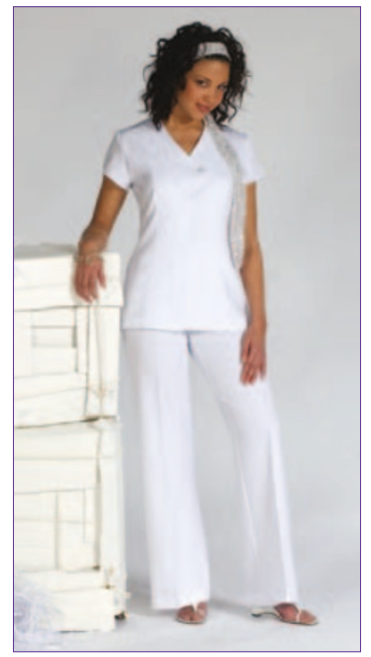

Exclusive to Florence Roby is the very latest linen weave fabric. It has been designed into the brand new Pathos Tunic, exquisitely finished with an elegant diamante button. Florence Roby's new linen weave fabric is also easy care and despite its linen appearance, requires minimal ironing. The classically cut Pathos Tunic is available in both black and white and when worn with the L34 Flared Trousers, completes an elegant suit. For more information visit www.uniformcollection.com or ring 01515482228 for a free colour brochure.

\section{Reminders by text}

A new text messaging service has been launched with the aim of reminding patients about their dental and medical appointments. The Messaging Centre (TMC) is a web-based text messaging platform designed to enhance business communications and continuity within Government healthcare departments, private GP practices, dental surgeries and hospitals or anyone with a company or personal mobile phone.

The service has been designed specifically to benefit industries such as healthcare and pharmaceutical industries by providing a corporate text messaging service that can dramatically improve business operations and communications and provide enhanced aftercare for outpatients.

The company was launched following research that found that around 15 million medical appointments were missed each year costing the Government and private healthcare industry millions in lost annual revenue.

The two-way text messages can be sent directly from many different

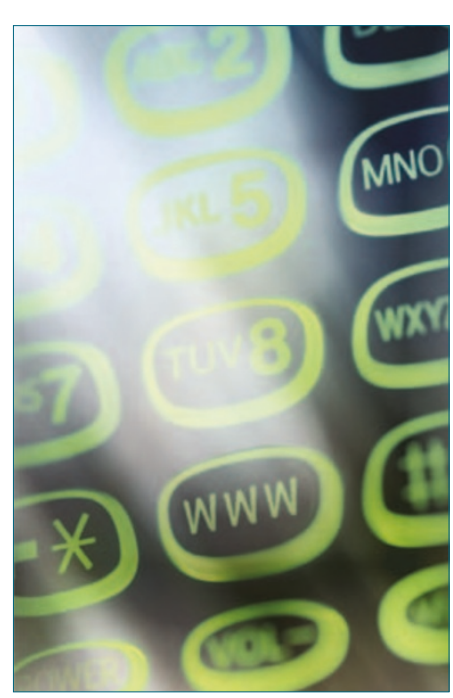
platforms such as PCs, wireless PDAs (Personal Digital Assistants), company or personal GSMs (Global Systems for Mobile communications).

The Messaging Centre features include accessing the system from any internet connected PC, no set up fee and no minimum monthly spend. There is also total control over who sends messages, no contract term and encryption ensures that your company's data are kept confidential. For more information visit www. tmcsms.co.uk.

\section{Give them gel}

Curaprox has announced the addition of Curasept Gel to its portfolio. This new oral hygiene product offers all the benefits of Curasept Mouth Rinse but in a gel.

Curasept Gel contains $0.5 \%$ chlorhexidine, is intended for shortterm, intensive use and offers effective protection against plaque and caries. The gel is alcohol-free and also has the ADS System which greatly reduces tooth discoloration and the unpleasant changes in taste perception associated with traditional chlorhexidine products. The product offers patients who would otherwise avoid chlorhexidine products the benefits of chlorhexidine in a more acceptable form. The makers add that Curasept Gel will help to prevent post-treatment and post-operative complications when used before or after dental treatment, including endodontic and implant procedures.

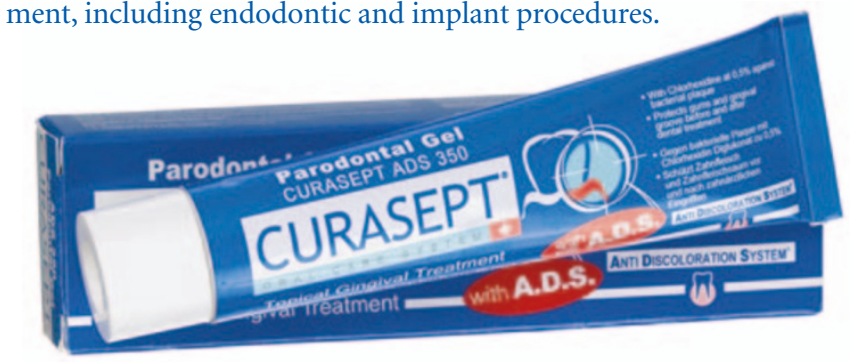

\section{All in one kit}

Dentsply Maillefer has launched the ProEndo Discovery Kit, an endo kit including materials and equipment required for cavity access, rotary root canal treatment, obturation and re-treatment.

The kit includes an X-Smart Endodontic motor with two autoclavable contra angles, three packs of NiTi ProTaper Universal treatment files, two packs of ProTaper Universal re-treatment files, re-treatment DMS IV solvent, new Senseus Flexofiles, Hedstroems and ProFinders, sealer, paper points and gutta percha points. A cavity access set is included and other accessories include glyde, mini-endo bloc and training blocs. Dentsply also provide training on all of the products, advising how they can be best used to benefit your practice and clinical procedures.

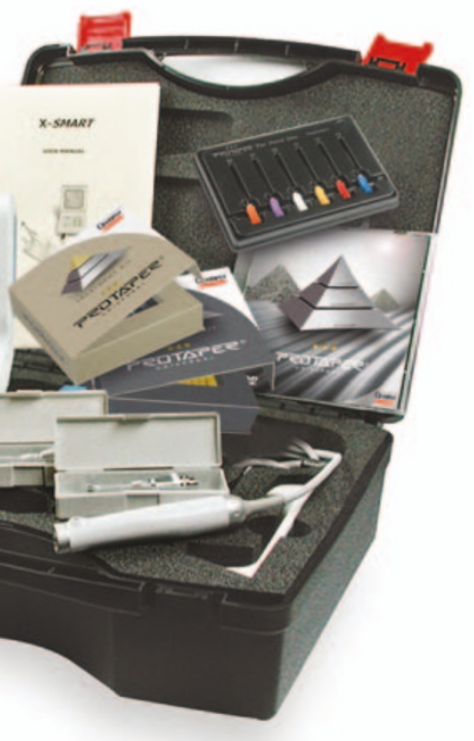

